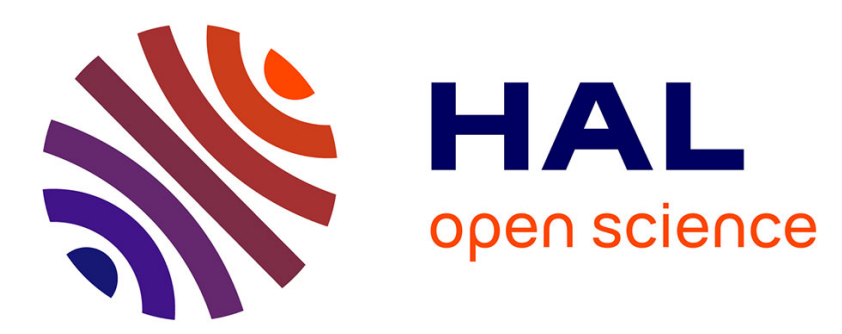

\title{
Retrieval of the physical properties of an anelastic solid half space from seismic data
}

Gaëlle Mesgouez, Arnaud Mesgouez, Erick Ogam, Thierry Scotti, Armand Wirgin

\section{- To cite this version:}

Gaëlle Mesgouez, Arnaud Mesgouez, Erick Ogam, Thierry Scotti, Armand Wirgin. Retrieval of the physical properties of an anelastic solid half space from seismic data. Journal of Applied Geophysics, 2013, 88, pp.70-82. 10.1016/j.jappgeo.2012.09.010 . hal-00657609

\section{HAL Id: hal-00657609 \\ https://hal.science/hal-00657609}

Submitted on 7 Jan 2012

HAL is a multi-disciplinary open access archive for the deposit and dissemination of scientific research documents, whether they are published or not. The documents may come from teaching and research institutions in France or abroad, or from public or private research centers.
L'archive ouverte pluridisciplinaire HAL, est destinée au dépôt et à la diffusion de documents scientifiques de niveau recherche, publiés ou non, émanant des établissements d'enseignement et de recherche français ou étrangers, des laboratoires publics ou privés. 


\title{
Retrieval of the source location and mechanical descriptors of a hysteretically-damped solid occupying a half space by full wave inversion of the the response signal on its boundary
}

\author{
Gaëlle Lefeuve-Mesgouez ${ }^{*}$ Arnaud Mesgouez †,

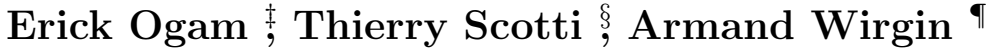

January 6, 2012

\begin{abstract}
The elastodynamic inverse problem treated herein can be illustrated by the simple acoustic inverse problem first studied by (Colladon, 1827): retrieve the speed of sound (C) in a liquid from the time $(\mathrm{T})$ it takes an acoustic pulse to travel the distance (D) from the point of its emission to the point of its reception in the liquid. The solution of Colladon's problem is obviously $\mathrm{C}=\mathrm{D} / \mathrm{T}$, and that of the related problem of the retrieval of the position of the source from $\mathrm{T}$ is $\mathrm{D}=\mathrm{CT}$. The type of questions we address in the present investigation, in which the liquid is a solid occupying a half space, $\mathrm{T}$ a complete signal rather than the instant at which it attains its maximum, and $\mathrm{C}$ a set of five parameters, are: how precise is the retrieval of $\mathrm{C}$ when $\mathrm{D}$ is known only approximately and how precise is the retrieval of $\mathrm{D}$ when $\mathrm{C}$ is plagued with error?
\end{abstract}

\footnotetext{
*Université d'Avignon et des Pays de Vaucluse, UMR EMMAH, Faculté des Sciences, F-84000 Avignon, France

${ }^{\dagger}$ Université d'Avignon et des Pays de Vaucluse, UMR EMMAH, Faculté des Sciences, F-84000 Avignon, France

${ }^{\ddagger}$ LMA, CNRS, UPR 7051, Aix-Marseille Univ, Centrale Marseille, F-13402 Marseille Cedex 20, France

${ }^{\S}$ LMA, CNRS, UPR 7051, Aix-Marseille Univ, Centrale Marseille, F-13402 Marseille Cedex 20, France

`LMA, CNRS, UPR 7051, Aix-Marseille Univ, Centrale Marseille, F-13402 Marseille Cedex 20, France
} 


\section{Contents}

1 General introduction $\quad 3$

1.1 Statement of the inverse problem . . . . . . . . . . . . . . . 3

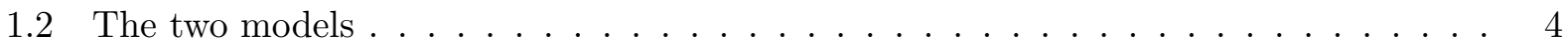

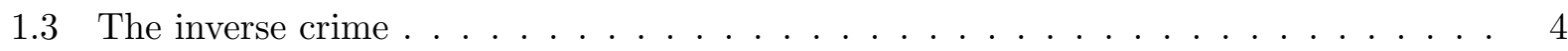

2 Ingredients of the data simulation and retrieval models 4

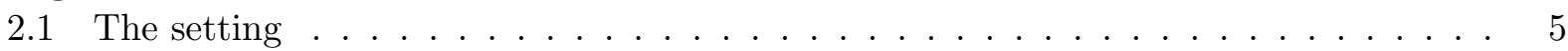

2.2 The boundary value problem $\ldots \ldots \ldots \ldots \ldots \ldots \ldots$

2.3 Material damping and complex body wave velocities . . . . . . . . . . . . 6

2.4 Plane wave field representations . . . . . . . . . . . . . . . . 7

2.5 Application of the boundary conditions to obtain the coefficients of the plane wave representations of the displacement field . . . . . . . . . . . . 8

2.6 Numerical issues concerning the computation of the transfer function . . . . . . . . 9

2.7 Vertical component of the displacement signal on the ground for vertical applied stress 11

2.8 Numerical issues concerning the computation of the response signal . . . . . . . . . 13

3 Ingredients and results of the inversion scheme $\quad 13$

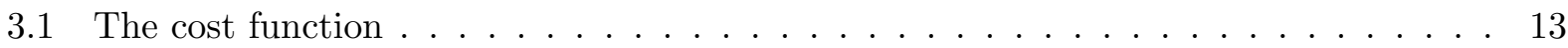

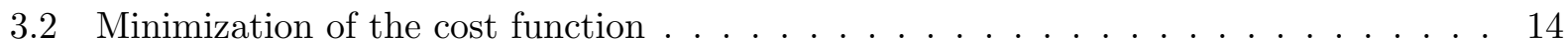

3.3 More on discordance and retrieval error . . . . . . . . . . . . . . . 15

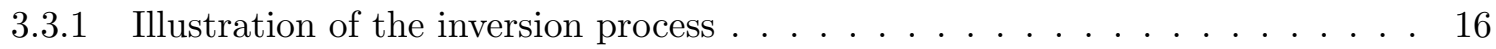

3.3.2 Retrieval error of $\rho \ldots \ldots \ldots \ldots \ldots \ldots$

3.3.3 Retrieval error of $\Re \lambda \ldots \ldots \ldots \ldots \ldots$

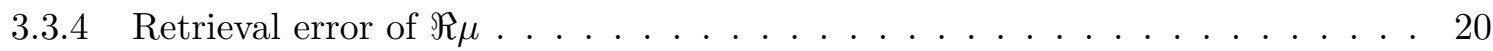

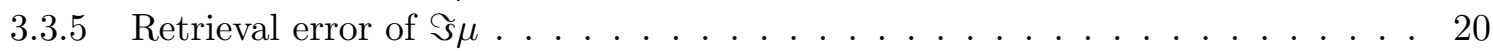

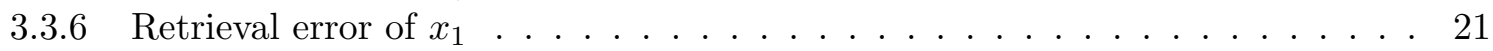

3.3.7 Comments on the tables relative to the retrieval errors resulting from the discordances . . . . . . . . . . . . . . . . . 21

4 Conclusion $\quad 21$ 


\section{General introduction}

We address herein the (inverse) problem of the retrieval of the source location and the material parameters of a homogeneous, isotropic, hysteretically-damped solid medium occupying a half space. The retrieval is accomplished by processing simulated (or measured, in any case, known) temporal response (the data) at a location on the (flat) bounding surface.

In the geophysical context (Tarantola,1986; Sacks \& Symes, 1987; Aki \& Richards, 1980), such problems concern earthquake (and underground nuclear explosion (Ringdal \& Kennett, 2001)) source localization (Billings et al., 1994; Thurber \& Rabinowitz, 2000; Michelini \& Lomax, 2004); Valentine \& Woodhouse, 2010) and underground mechanical descriptor retrieval (Tarantola, 1986), and are often solved (Zhang \& Chan, 2003; Lai et al., 2002) by inverting the times of arrival (TOAI) of body (Kikuchi \& Kanamori, 1982) and surface (Xia et al., 1999) waves in the displacement signal at one or several points on the boundary of the medium. This approach requires the prior identification of the maxima or minima (or other signatures) of the signal corresponding to these times of arrival and thus is fraught with ambiguity, especially when body wave and surface wave times of arrivals are close as at small offsets (Bodet 2005; Foti et al., 2009) or when many surface waves (e.g., corresponding to generalized Rayleigh modes) contribute in a complex manner to the time domain response, as when the underlying medium is multilayered (Aki \& Richards, 1980; Foti et al., 2009).

What appears to be less ambiguous is to employ most (or all) of the information in the signal (or of its spectrum (Mora, 1987; Sun \& McMechan, 1992; Pratt, 1999; Virieux \& Operto, 2009; De Barros et al, 2010; Dupuy, 2011)) in the inversion process (full waveform inversion, FWI) rather than a very small fraction of the signal (as in the TOAI) methods.

We shall determine, in the context of the simplest canonical problem, to what extent a time domain FWI method enables the retrieval of either the source location or of one of the mechanical descriptors: (real) mass density, and (complex) Lamé parameters of the medium, when the remaining parameters are not well-known a priori. This type of study was initiated in (Buchanan et al., 2002; Chotiros, 2002), and continued in such works as (Scotti \& Wirgin, 2004; Buchanan et al., 2011; Dupuy, 2011).

\subsection{Statement of the inverse problem}

As we shall see hereafter, the data takes the form of a response signal (to a dynamic load, over a temporal window $\left[t_{d}, t_{f}\right]$, sampled at $N_{t}$ instants) which is a double integral $U$ (over nondimensional wavenumber $\xi$ and frequency $f$ ) depending on certain physical and geometrical scalar parameters of the scattering structure and of the solicitation. These parameters $p_{1}, p_{2}, \ldots p_{K}, \ldots, p_{N}$ form the set $\mathbf{p}$.

The forward scattering problem is to determine $U(\mathbf{p}, t)$ for different combinations of $p_{1}, p_{2}, \ldots, p_{N}$.

Our inverse scattering problem is to recover one or several of the parameters $p_{1}, p_{2}, \ldots, p_{N}$ from data pertaining to the signal $\left\{U(\mathbf{p}, t) ; t \in\left[t_{d}, t_{f}\right]\right\}$. $\left(x_{1}, 0\right)$ are the cartesian coordinates of the position of the receiver on the ground and $(0,0)$ the position of the emitter, also on the ground, of the probe signal.

The present study is restricted to the case in which only a single parameter $p_{K}$ of $\mathbf{p}$ is retrieved at a time, the other parameters of $\mathbf{p}$ being assumed to be more or less well-known (Aki \& Richards, 
1980). Hereafter, we adopt the notation: $\mathbf{q}:=\mathbf{p}-p_{K}$.

In fact, we are most interested herein in evaluating to what extent the precision of retrieval of $p_{K}$ depends on the degree of a priori knowledge of the other parameters of $\mathbf{p}$.

\subsection{The two models}

In order to carry out an inversion of a set of data one must dispose of a model of the physical process he thinks is able to generate the data. We term this model, the retrieval model, or RM. The RM is characterized by: 1) the mathematical/numerical ingredient(s) (MNI) and 2) the physical/geometrical and numerical parameters to which the model appeals. The physical/geometrical parameters of the RM form the set $\mathbf{P}$, whereas the numerical parameters of the RM can be grouped into a set which we call $\mathbf{N}$.

When, as in the present study, the (true) data is not the result of a measurement, it must be generated (simulated), again with the help of a model of the underlying physical process which is thought to be able to give rise to the true data. We term this model, the data simulation model, or $S M$. The SM, like the RM, is characterized by two essential ingredients: the mathematical/numerical ingredient(s) (MNI) and the physical/geometrical and numerical parameters to which the model appeals. The physical/geometrical parameters of the SM form none other than the set $\mathbf{p}$, whereas the numerical parameters of the SM can be grouped into a set which we call $\mathbf{n}$.

\subsection{The inverse crime}

In the present study, as in many other inverse problem investigations, the MNI of the RM is chosen to be the same as the MNI of the SM. In this case, when the values of all the parameters of the set $\mathbf{P}$ are strictly equal to their counterparts in the set $\mathbf{p}$ and the values of all the parameters of the set $\mathbf{N}$ are strictly equal to their counterparts in the set $\mathbf{n}$, the response computed via the RM will be identical to the response computed via the SM.

This so-called 'trivial' result, which is called the 'inverse crime' in the inverse problem context (Colton \& Kress, 1992), has a corollary (Wirgin, 2004): when the values of all the parameters, except $P_{K}$ of the set $\mathbf{P}$ are strictly equal to their counterparts in the set $\mathbf{p}$ and the values of all the parameters of the set $\mathbf{N}$ are strictly equal to their counterparts in the set $\mathbf{n}$, then the inversion will give rise to at least one solution, $P_{K}=p_{K}$.

This eventuality is highly improbable in real-life, in that one usually has only a vague idea a priori of the value of at least one of the parameters of the set $\mathbf{p}$. This is the reason why, in the present study, we take explicitly in account this imprecision, with the added benefit of avoiding the inverse crime.

\section{Ingredients of the data simulation and retrieval models}

As mentioned previously, herein the two models SM and RM are assumed to be identical as to their mathematical/numerical ingredients (MNI) and the nature and number of involved physi$\mathrm{cal} /$ geometrical parameters. We now proceed to describe these MNI. 


\subsection{The setting}

Space is divided into two half spaces: $\Omega$ (termed hereafter underground), and $\mathbb{R}^{3} \backslash \Omega$. The medium $M$ occupying $\Omega$, is a linear, isotropic, homogeneous, hysteretically-damped solid and the medium occupying $\mathbb{R}^{3} \backslash \Omega$ is the vacumn.

$M$ is associated with: $\rho$, its mass density and $\lambda, \mu$, its Lamé constitutive parameters. Due to the homogeneous, isotropic nature of $M, \rho, \lambda^{\prime}, \lambda^{\prime \prime}, \mu^{\prime}$ and $\mu^{\prime \prime}$ are real, scalar constants, with the understanding that primed quantities are related to the real part and double primed quantitites to the imaginary part of a complex parameter.

Let $G$, termed hereafter ground, designate the flat horizontal interface between these two half spaces and $\boldsymbol{\nu}$ be the unit vector normal to $G$.

Let $t$ be the time, $\mathbf{x}:=\left(x_{1}, x_{2}, x_{3}\right)$ the vector from the origin (located on $G$ ) to a generic point in space, and $x_{m}$ a cartesian coordinate, such that $\boldsymbol{\nu}=(0,0,1)$. Let $\mathbf{U}=\left\{U_{m}(\mathbf{x}, t) ; m=1,2,3\right\}$ designate the displacement in the medium, with spatial derivatives $U_{k, l}:=\partial U_{k} / \partial x_{l}$.

The medium is solicited by stresses applied on the portion $G^{a}$ of $G$. Other than on $G^{a}$, the boundary $G$ is stress-free. In addition, we assume that: (1) $G^{a}$ is an infinitely long (along $x_{2}$ ) strip located between $x_{1}=-a$ and $x_{1}=a$ and (2) the applied stresses are uniform, so that the stresses and the displacement $\mathbf{U}$ depend only on $x_{1}$ and $x_{3}$, i.e., the problem is two-dimensional. Thus, from now on, the focus is on what happens in the sagittal $\left(x_{1}-x_{3}\right)$ plane (see fig.1) and on the linear traces $\Gamma$ of $G$ and $\Gamma^{a}$ of $G^{a}$. Moreover, the vector $\mathbf{x}$ is now understood to evolve in the sagittal plane, i.e., $\mathbf{x}=\left(x_{1}, 0, x_{3}\right)$ and all derivatives of displacement with respect to $x_{2}$ are nil.

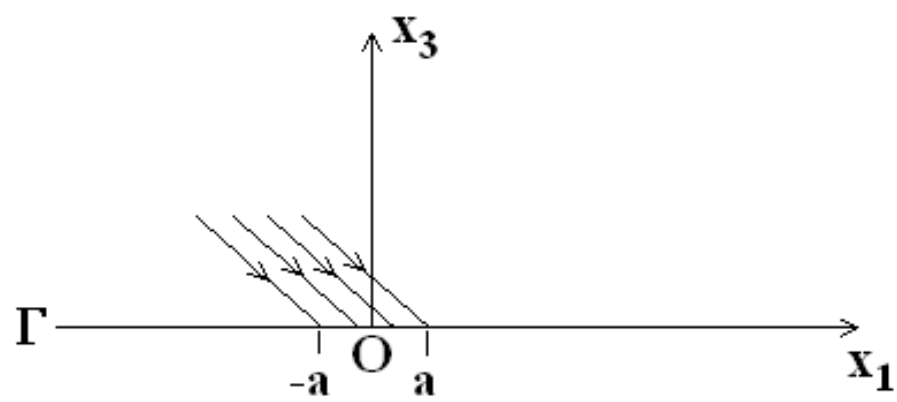

$\Omega$

Figure 1: Description of the problem in the sagittal plane.

\subsection{The boundary value problem}

By expanding $\mathbf{U}$ in a Fourier integral (with $\omega$ the angular frequency):

$$
\mathbf{U}(\mathbf{x}, t)=\int_{-\infty}^{\infty} \mathbf{u}(\mathbf{x}, \omega) \exp (-i \omega t) d \omega
$$


the Navier equations (Eringen \& Suhubi, 1975) become (with the Einstein index summation convention):

$$
(\lambda+\mu) u_{k, k l}+\mu u_{l, k k}+\rho \omega^{2} u_{l}=0,
$$

wherein

$$
\lambda:=\lambda^{\prime}-i \omega \lambda^{\prime \prime} \quad, \quad \mu:=\mu^{\prime}-i \omega \mu^{\prime \prime} .
$$

The boundary conditions are:

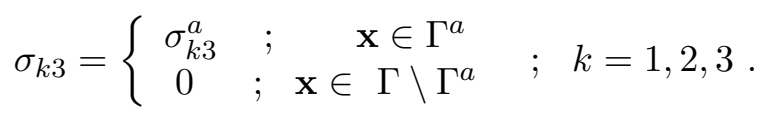

or,

$$
\begin{aligned}
& \mu\left(u_{1,3}+u_{3,1}\right)=\left\{\begin{array}{ccc}
\sigma_{13}^{a} & ; & \mathbf{x} \in \Gamma^{a} \\
0 & ; & \mathbf{x} \in \Gamma \backslash \Gamma^{a}
\end{array},\right. \\
& \mu u_{2,3}=\left\{\begin{array}{c}
\sigma_{23}^{a} \quad ; \quad \mathbf{x} \in \Gamma^{a} \\
0 \quad ; \quad \mathbf{x} \in \Gamma \backslash \Gamma^{a},
\end{array}\right. \\
& \lambda u_{1,1}+(\lambda+2 \mu) u_{3,3}=\left\{\begin{array}{ccc}
\sigma_{33}^{a} & ; & \mathbf{x} \in \Gamma^{a} \\
0 & ; & \mathbf{x} \in \Gamma \backslash \Gamma^{a}
\end{array},\right.
\end{aligned}
$$

wherein $\sigma_{k l}$ are the components of the space-frequency domain stress tensor.

Finally, the displacement in the solid is subjected to the radiation condition

$$
u_{m}(\mathbf{x}, \omega) \sim \text { attenuated waves } ; \mathbf{x} \in \Omega, x_{3} \rightarrow-\infty .
$$

\subsection{Material damping and complex body wave velocities}

We can rewrite $\mu$ and $\lambda$ as

$$
\mu=\mu^{\prime}\left(1-i \omega \frac{\mu^{\prime \prime}}{\mu^{\prime}}\right) \quad, \quad \lambda=\lambda^{\prime}\left(1-i \omega \frac{\lambda^{\prime \prime}}{\lambda^{\prime}}\right) .
$$

The case of hysteretic damping (Molenkamp \& Smith, 1980; Mesgouez, 2005), assumed in this study, corresponds to

$$
\beta_{\mu}:=\omega \frac{\mu^{\prime \prime}}{\mu^{\prime}}, \beta_{\lambda}:=\omega \frac{\lambda^{\prime \prime}}{\lambda^{\prime}},
$$

whence

$$
\mu=\mu^{\prime}\left(1-i \beta_{\mu}\right) \quad, \quad \lambda=\lambda^{\prime}\left(1-i \beta_{\lambda}\right),
$$

wherein $\beta_{\mu}$ and $\beta_{\lambda}$ are constants (i.e., with respect to frequency $\omega$ ). This implies that $\frac{\mu^{\prime \prime}}{\mu^{\prime}}=\frac{\beta_{\mu}}{\omega}$ and/or $\frac{\lambda^{\prime \prime}}{\lambda^{\prime}}=\frac{\beta_{\lambda}}{\omega}$, which means that $\mu^{\prime \prime}$ and/or $\mu^{\prime}$ depend on the frequency and $\lambda^{\prime \prime}$ and/or $\lambda^{\prime}$ depend on the frequency.

A typical solution of (2) is of the (plane wave) form:

$$
u_{l}(\mathbf{x}, \omega)=A_{l}(\omega) \exp \left(i k_{m} x_{m}\right)
$$

wherein

$$
k_{m} k_{m}=k^{2}=\frac{\omega^{2}}{c^{2}}
$$


and one finds the three eigenvalues:

$$
\begin{gathered}
c=c_{1}=c_{2}=\sqrt{\mu / \rho}=c_{S}, \\
c=c_{3}=\sqrt{(\lambda+2 \mu) / \rho}=c_{P} .
\end{gathered}
$$

which are recognized to be the velocities of the transverse (shear, Secondary) and longitudinal (compressional, Primary) bulk waves in the damped solid medium.

These velocities, and in particular, $c_{S}$, are complex, i.e.,

$$
c_{S}=c_{S}^{\prime}-i c_{S}^{\prime \prime} .
$$

We require

$$
\Re c_{S}=c_{S}^{\prime} \geq 0,
$$

due to the fact that the body wave velocity is positive in an elastic (i.e., non-lossy medium). We have

$$
k_{S}=k_{S}^{\prime}+i k_{S}^{\prime \prime}=\frac{\omega}{c_{S}}=\frac{\omega}{c_{S}^{\prime}-i c_{S}^{\prime \prime}}=\frac{\omega c_{S}^{\prime}+i \omega c_{S}^{\prime \prime}}{\left\|c_{S}\right\|^{2}},
$$

from which we see that in order for $\Im k_{S}=k_{S}^{\prime \prime} \geq 0$, we must have

$$
\Im c_{S}=-c_{S}^{\prime \prime} \leq 0 \text {. }
$$

In the same manner we can show that

$$
\Im c_{P}=-c_{P}^{\prime \prime} \leq 0
$$

\subsection{Plane wave field representations}

By employing the Helmholtz decomposition, the gauge condition and the radiation condition to (2), we obtain the following plane wave representations of the displacement

$$
\begin{gathered}
u_{1}(\mathbf{x}, \omega)=\int_{-\infty}^{\infty}\left[A_{1}^{-}\left(\omega, k_{1}\right) k_{1} E_{P}^{-}\left(\mathbf{x}, \omega, k_{1}\right)+A_{2}^{-}\left(\omega, k_{1}\right) k_{3 S} E_{S}^{-}\left(\mathbf{x}, \omega, k_{1}\right)\right] d k_{1} \\
u_{2}(\mathbf{x}, \omega)=\int_{-\infty}^{\infty} A_{3}^{-}\left(\omega, k_{1}\right) k_{S} E_{S}^{-}\left(\mathbf{x}, \omega, k_{1}\right) d k_{1} \\
u_{3}(\mathbf{x}, \omega)=\int_{-\infty}^{\infty}\left[-A_{1}^{-}\left(\omega, k_{1}\right) k_{3 P} E_{P}^{-}\left(\mathbf{x}, \omega, k_{1}\right)+A_{2}^{-}\left(\omega, k_{1}\right) k_{1} E_{S}^{-}\left(\mathbf{x}, \omega, k_{1}\right)\right] d k_{1},
\end{gathered}
$$

wherein, for $k_{1} \in \mathbb{R}$,

$$
\begin{aligned}
& k_{3 P}=\sqrt{\kappa_{3 P}}=\sqrt{\left(k_{P}\right)^{2}-\left(k_{1}\right)^{2}} ; \\
& \Re k_{3 P} \geq 0, \Im k_{3 P} \geq 0 \text { when } \Im \kappa_{3 P} \geq 0, \Im k_{3 P}<0 \text { when } \Im \kappa_{3 P}<0 \text {; for } \omega \geq 0, \\
& k_{3 S}=\sqrt{\kappa_{3 S}}=\sqrt{\left(k_{S}\right)^{2}-\left(k_{1}\right)^{2}} ; \\
& \Re k_{3 S} \geq 0, \Im k_{3 S} \geq 0 \text { when } \Im \kappa_{3 S} \geq 0, \Im k_{3 S}<0 \text { when } \Im \kappa_{3 S}<0 \text {; for } \omega \geq 0,
\end{aligned}
$$


and

$$
E_{P}^{-}:=\exp \left[i\left(k_{1} x_{1}-k_{3 P} x_{3}\right)\right], \quad E_{S}^{-}:=\exp \left[i\left(k_{1} x_{1}-k_{3 S} x_{3}\right)\right] .
$$

The previous choices of signs of the real and imaginary parts of $k_{3 P}$ and $k_{3 S}$ for $\omega \geq 0$ in (24)-(25) were conventional. The question arises, due to the fact that the time domain response is a Fourier integral involving negative frequencies as well as zero and positive frequencies, as to what signs to choose when $\omega<0$. The answer is provided by the requirement that the physical space time-domain displacement field $u_{j}(\mathbf{x}, t)$ be real, and is easily shown to lead to:

$$
\begin{aligned}
& \Re k_{3 P}(\omega) \geq 0, \Im k_{3 P}(\omega)<0 \text { when } \Im \kappa_{3 P} \geq 0, \Im k_{3 P} \geq 0 \text { when } \Im \kappa_{3 P}<0 ; \text { for } \omega<0, \\
& \Re k_{3 S}(\omega) \geq 0, \Im k_{3 S}(\omega)<0 \text { when } \Im \kappa_{3 S} \geq 0, \Im k_{3 S} \geq 0 \text { when } \Im \kappa_{3 S}<0 ; \text { for } \omega<0 .
\end{aligned}
$$

Eqs. (21)-(23) express the fact that 2D fields are composed of:

a) in-(sagittal) plane motion, embodied by a sum of $\mathrm{P}$ (for pressure)-polarized and SV (for shear vertical) -polarized plane waves, and

b) out-of-(sagittal) plane motion, embodied by a sum of SH (for shear horizontal) -polarized plane waves.

\subsection{Application of the boundary conditions to obtain the coefficients of the plane wave representations of the displacement field}

From now on, we restrict the discussion to in-plane motion, so that the introduction of the plane wave representations into the boundary conditions yields:

$$
\begin{gathered}
A_{1}^{-}\left[-2 i \mu k_{1} k_{3 P}\right]+A_{2}^{-}\left[i \mu\left(k_{1}^{2}-k_{3 S}^{2}\right)\right]=S_{13}^{a} \quad ; \quad \forall k_{1} \in \mathbb{R}, \\
A_{1}^{-}\left[-i \mu\left(k_{1}^{2}-k_{3 S}^{2}\right)\right]+A_{2}^{-}\left[-2 i \mu\left(k_{1} k_{3 S}\right)\right]=S_{33}^{a} \quad ; \quad \forall k_{1} \in \mathbb{R} .
\end{gathered}
$$

wherein:

$$
S_{k l}^{a}\left(x_{1}, 0, \omega, k_{1}\right):=\int_{-a}^{a} \sigma_{k l}^{a}\left(x_{1}, 0, \omega\right) \exp \left(-i k_{1} x_{1}\right) d x_{1} \quad ; \quad \forall k_{1} \in \mathbb{R} .
$$

On account of the uniform strip-like character of the solicitation, we have:

$$
\sigma_{j 3}^{a}\left(x_{1}, 0, \omega\right)=\mathcal{P}_{j} \mathcal{H}(\omega) ; x_{1} \in[-a, a],
$$

wherein $\mathcal{P}_{j}$ are prescribed constants, and $\mathcal{H}(\omega)$ is the spectrum of applied stress, such that

$$
\mathcal{H}(-\omega)=\mathcal{H}(\omega)
$$

It ensues that

$$
S_{j 3}^{a}\left(x_{1}, 0, \omega, k_{Q} \xi\right)=\mathcal{P}_{j} \mathcal{H}(\omega) \int_{-a}^{a} \exp \left(-i k_{Q} \xi x_{1}\right) d x_{1}=2 a \mathcal{P}_{j} \mathcal{H}(\omega) \operatorname{sinc}(\xi \omega A),
$$

wherein $\operatorname{sinc}(x):=\frac{\sin x}{x}$ and

$$
A:=\frac{a}{c_{Q}}, \quad X_{j}:=\frac{x_{j}}{c_{Q}}
$$


We now make the change of variables

$$
k_{1}=k_{Q} \xi \text { with } k_{Q}=\frac{\omega}{c_{Q}} .
$$

$c_{Q}$ is a reference velocity with no particular characteristics other than

$$
\Re c_{Q}>0, \Im c_{Q}=0
$$

and is otherwise arbitrary. Then

$$
k_{3 P, S}:=k_{Q} \chi_{P, S},
$$

wherein

$$
\begin{gathered}
\chi_{P}(\xi)=\sqrt{r_{P}^{2}-\xi^{2}}, \quad \chi_{S}(\xi)=\sqrt{r_{S}^{2}-\xi^{2}}, \\
r_{P}=\frac{c_{Q}}{c_{P}}, \quad r_{S}=\frac{c_{Q}}{c_{S}},
\end{gathered}
$$

and we adopt the same sign convention for $\chi_{P}$ and $\chi_{S}$ as for $k_{P}$ and $k_{S}$ respectively.

By finally restricting our attention to vertical motion (i.e, $u_{3}$ ) in response to vertical stress (i.e., only $\mathcal{P}_{3} \neq 0$ ) we obtain (since $\chi_{P, S}(-\xi)=\chi_{P, S}(\xi)$ ), by solving $(29)-(30)$ for $A_{1}^{-}$and $A_{3}^{-}$:

$$
\begin{aligned}
& u_{3}(\mathbf{x}, \omega)=\frac{4 i a \mathcal{P}_{3} \mathcal{H}(\omega)}{\mu} \times \\
& \int_{0}^{\infty}\left[-\chi_{P}\left[\xi^{2}-\chi_{S}^{2}\right] \exp \left(-i \chi_{P} \omega X_{3}\right)+2 \xi^{2} \chi_{P} \exp \left(-i \chi_{S} \omega X_{3}\right)\right] \frac{\operatorname{sinc}(\xi \omega A) \cos \left(\xi \omega X_{1}\right)}{4 \xi^{2} \chi_{P} \chi_{S}+\left[\xi^{2}-\chi_{S}^{2}\right]} d \xi,
\end{aligned}
$$

which is the space-frequency solution to the forward problem of the prediction of the vertical component of displacement response to a uniform vertical strip load on the boundary of the half space.

\subsection{Numerical issues concerning the computation of the transfer function}

On the ground (which is where the data is collected), (41) tells us that

$$
u_{3}\left(x_{1}, 0, \omega\right)=\mathcal{H}(\omega) \mathcal{T}\left(x_{1}, 0, \omega\right),
$$

wherein $\mathcal{T}\left(x_{1}, 0, \omega\right)$ is the transfer function

$$
\mathcal{T}\left(x_{1}, 0, \omega\right)=\int_{0}^{\infty} \frac{N\left(x_{1}, 0, \xi, \omega\right)}{D(\xi)} d \xi
$$

with

$$
\begin{aligned}
& N\left(x_{1}, 0, \xi, \omega=i \mathcal{Q}_{3} r_{S}^{2} \chi_{P}(\xi, \omega) \operatorname{sinc}(\xi \omega A) \cos \left(\xi \omega X_{1}\right),\right. \\
& D(\xi)=4 \xi^{2} \chi_{P}(\xi, \omega) \chi_{S}(\xi, \omega)+\left[\xi^{2}-\left(\chi_{S}(\xi, \omega)\right)^{2}\right], \quad \mathcal{Q}_{j}:=\frac{4 a \mathcal{P}_{j}}{\mu} .
\end{aligned}
$$

Various strategies have been devised (Fu, 1947; Apsel \& Luco, ,1983; Xu \& Mal, 1987; Stam, 1990; Chen \& Zhang, 2001; Park \& Kausel, 2004; Groby, 2005, Groby \& Wirgin 2005; Mesgouez 
\& Lefeuve-Mesgouez, 2009) to compute such integrals, many of which take specific account of the possible (generally-complex) solutions of $D(\xi)=0$ (the equation for the Rayleigh mode eigenvalues) close (all the more so, the smaller is the attenuation in the solid medium) to the real $\xi$ axis, but herein we make the simpler choice of direct numerical quadrature.

To do this, we first make the approximation

$$
\mathcal{T}\left(x_{1}, 0, \omega\right) \approx \int_{\xi_{d}}^{\xi_{f}} \frac{N\left(x_{1}, 0, \xi, \omega\right)}{D(\xi)} d \xi
$$

with $\xi_{d}$ being close to 0 and $\xi_{f}$ being as large as (is economically) possible. The second step is to replace the integral by any standard numerical quadrature scheme, i.e.,

$$
\mathcal{T}\left(x_{1}, 0, \omega\right) \approx \varepsilon_{\xi} \sum_{n=1}^{N_{\xi}} w_{n} \frac{N\left(x_{1}, 0, \xi_{n}, \omega\right)}{D\left(\xi_{n}\right)},
$$

wherein, for instance, $\xi_{n}=\xi_{d}+(n-1) \varepsilon_{\xi}, \varepsilon_{\xi}=\left(\xi_{f}-\xi_{d}\right) /\left(N_{\xi}-1\right)$ and the $w_{n}$ are the weights associated with the chosen quadrature scheme.

In fact, we evaluated the rectangular, trapezoidal, Simpson and various Matlab functions, and finally settled for the Simpson quadrature technique.

The principal problem is then the proper choice of $\xi_{d}, \xi_{f}$ and $N_{\xi}$. This was done by sequential variation of these three numerical parameters until the achievement of stabilization of the computational result. The optimal set $\boldsymbol{\xi}=\left\{\xi_{d}, \xi_{f}, N_{\xi}\right\}$ was then the one that first enabled the achievement of this stabilization.

An alternative to this method is possible when supposedly-accurate reference results (as obtained, for instance, by an adaptive Filon integration scheme (Chen \& Zhang, 2001)) are available. In this case, the choice of optimal numerical parameters is made on the basis of a minimal norm, the norm being (for instance)

$$
\mathcal{N}\left(x_{1}, 0, \boldsymbol{\xi}\right):=\int_{f_{d}}^{f_{f}}\left\|\mathcal{T}_{\text {ref }}\left(x_{1}, 0,2 \pi f\right)-\mathcal{T}_{\text {trial }}\left(x_{1}, 0,2 \pi f, \boldsymbol{\xi}\right)\right\|^{2} d f,
$$

wherein $f=\omega / 2 \pi$ is the frequency, whereas $\mathcal{T}_{\text {ref }}$ is the reference solution and $\mathcal{T}_{\text {trial }}$ the solution with trial numerical parameters $\xi_{d}, \xi_{f}$ and $N_{\xi}$.

It is important to underline the fact that in the inverse problem context, it is not crucial to obtain a perfectly-accurate solution of the forward problem (in fact, one often deliberately adds noise to 'spoil' the inverse crime and/or to simulate measurement error), since the same solution is employed for the simulation of data and for a retrieval model, both of these being fraught, in realworld situations, with errors of all sorts (noise, uncertainty of various physical and/or geometrical parameters intervening in: the measurement or simulation of data, and the retrieval model of the displacement on the ground). Moreover, as shown in (Wirgin, 2004), the success of an inversion is largely due to the extent to which the retrieval model accounts for all features of the data, and when the data is simulated, the ideal situation (i.e., in which the inverse crime is committed) is obtained by employing the same model for the retrieval as the one employed for the simulation of data, this being true whether this model gives a true picture of reality or not. 


\subsection{Vertical component of the displacement signal on the ground for vertical applied stress}

Recall that the relation between the displacement spectrum $\mathbf{u}(\mathbf{x}, \omega)$ and the displacement signal $\mathbf{u}(\mathbf{x}, t)$ is

$$
\mathbf{U}(\mathbf{x}, t)=\int_{0}^{\infty}[\mathbf{u}(\mathbf{x},-\omega) \exp (i \omega t)+\mathbf{u}(\mathbf{x}, \omega) \exp (-i \omega t)] d \omega .
$$

This leads us to inquire as to the expression of $u_{3}\left(x_{1}, 0, \omega\right)$, which, on account of (41), (33), and the assumption of hysteretic damping (i.e., $r_{P, S}$ do not depend on $\omega$, making $D$ independent of the frequency, i.e., the Rayleigh modes are not dispersive in a hysteretically-damped or elastic medium), reads

$$
u_{3}\left(x_{1}, 0,-\omega\right)=i Q_{3} \mathcal{H}(\omega) \int_{0}^{\infty} r_{S}^{2} \chi_{P}(\xi,-\omega) \frac{\operatorname{sinc}(-\xi \omega A) \cos \left(\xi \omega X_{1}\right)}{D(\xi)} d \xi
$$

or, on account of the previous assumptions $\chi_{P, S}(\xi,-\omega)=\overline{\chi_{P, S}(\xi, \omega)}$,

$$
u_{3}\left(x_{1}, 0,-\omega\right)=\overline{u_{3}\left(x_{1}, 0, \omega\right)} \text {. }
$$

Consequently

$$
U_{3}\left(x_{1}, 0, t\right)=2 \Re \int_{0}^{\infty} u_{3}\left(x_{1}, 0, \omega\right) \exp (-i \omega t) d \omega .
$$

The applied stress signal on the boundary can generally be expressed as

$$
\sigma_{33}^{a}\left(x_{1}, 0, t\right)=\mathfrak{F}\left(x_{1}\right) \mathfrak{H}(t) .
$$

associated with the spectrum

$$
\sigma_{33}^{a}\left(x_{1}, 0, \omega\right)=\mathfrak{F}\left(x_{1}\right) \mathcal{H}(\omega) .
$$

The uniform nature of the applied stress was previously shown to translate to $\mathfrak{F}\left(x_{1}\right)=\mathcal{P}_{3}=$ constant. Here we dwell on $\mathfrak{H}(t)$ and its Fourier transform.

We choose the truncated sinusoidal impulsive excitation

$$
\mathfrak{H}(t)=H_{0} \sin \left(\omega_{0} t\right)\left[H(t)-H\left(t-2 t_{1}\right],\right.
$$

wherein $H_{0}, \omega_{0}$ and $t_{1}$ are (chosen) constants and $H(t)$ is the Heaviside function $(=0$ for $t<0$ and $=1$ for $t>0$ ). Since we want the pulse to take the form of a half period of a sinusoid in its non-vanishing portion, we take

$$
t_{1}=\frac{\pi}{2 \omega_{0}} .
$$

Then

$$
\sigma_{33}^{a}\left(x_{1}, 0, \omega\right)=\frac{\mathfrak{F}\left(x_{1}\right) H_{0} t_{1}}{2 \pi i} \exp \left(i \omega t_{1}\right)\left[\operatorname{sinc}\left(\left(\omega+\omega_{0}\right) t_{1}\right)+\operatorname{sinc}\left(\left(\omega-\omega_{0}\right) t_{1}\right)\right],
$$

whence

$$
\mathcal{H}(\omega)=\frac{H_{0} t_{1}}{2 \pi i} \exp \left(i \omega t_{1}\right)\left[\operatorname{sinc}\left(\left(\omega+\omega_{0}\right) t_{1}\right)+\operatorname{sinc}\left(\left(\omega-\omega_{0}\right) t_{1}\right)\right] .
$$

An example of this type of solicitation signal is given in fig. 2 . 

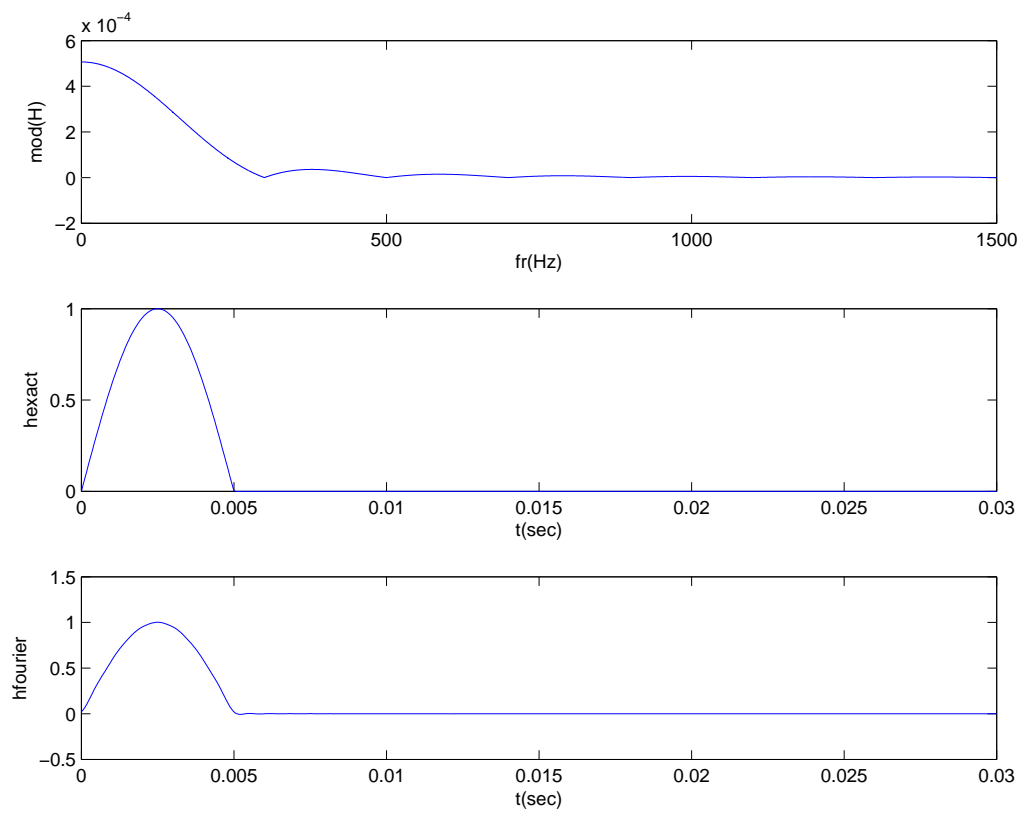

Figure 2: Modulus of the spectrum (top panel) and exact time (middle panel) domain representations of a half-sinusoidal pulse for which $\omega_{0}=200 \pi \mathrm{rad}, t_{1}=0.0025 \mathrm{~s}$. The bottom panel depicts the Fourier integral reconstruction of the pulse from its spectrum. 


\subsection{Numerical issues concerning the computation of the response signal}

We found in (51) that the response signal takes the form

$$
U_{3}\left(x_{1}, 0, t\right)=\int_{0}^{\infty} \mathcal{U}_{3}\left(x_{1}, 0, f\right) d f
$$

with $\omega=2 \pi f$ and

$$
\mathcal{U}_{3}\left(x_{1}, 0, f\right)=4 \pi \Re\left[u_{3}\left(x_{1}, 0,2 \pi f\right) \exp (-i 2 \pi f t)\right] .
$$

Once again, we make the simple choice of direct numerical quadrature by first adopting the approximation

$$
U_{3}\left(x_{1}, 0, t\right) \approx \int_{f_{d}}^{f_{f}} \mathcal{U}_{3}\left(x_{1}, 0, f\right) d f
$$

with $f_{d}$ being close to 0 and $f_{f}$ being as large as (is economically) possible. Actually, the choice of $f_{d}$ and $f_{f}$ is dictated a minima by the requirement that the significant portion of the spectrum of the excitation signal be accounted for.

The second step is to replace the integral by any standard numerical quadrature scheme, i.e.,

$$
U_{3}\left(x_{1}, 0, t\right) \approx \varepsilon_{f} \sum_{n=1}^{N_{f}} W_{n} \mathcal{U}_{3}\left(x_{1}, 0, f_{n}\right),
$$

wherein, for instance, $f_{n}=f_{d}+(n-1) \varepsilon_{f}, \varepsilon_{f}=\left(f_{f}-f_{d}\right) /\left(N_{f}-1\right)$ and the $W_{n}$ are the weights associated with the chosen quadrature scheme.

In fact, we settled for the Simpson quadrature technique and chose $f_{d}, f_{f}$ and $N_{f}$ as were previously chosen the numerical parameters in the computation of the transfer function.

Our numerical results for the forward problem space-time domain response were found to be in qualitative (if not quantitative) agreement with those of (Eringen \& Suhubi, 1975; Virieux, 1986; Pratt, 1990; Jones \& Petyt, 1991; Ma \& Lee, 2000; Park \& Kausel, 2004; Kausel, 2006), and those obtained by a method described in (Chen \& Zhang, 2001).

\section{Ingredients and results of the inversion scheme}

Recall that the to-be-retrieved parameters are: $p_{1}=\rho, p_{2}=\Re \lambda, p_{3}=\Im \lambda, p_{4}=\Re \mu, p_{5}=\Im \mu$, $p_{6}=x_{1}$. The other parameters, $\mathcal{P}, t_{1}, f_{0}=\omega / 2 \pi$ and $a$, relative to the solicitation, are assumed to be perfectly well-known a priori.

\subsection{The cost function}

Inversion is the process by which data (input to the process) is analyzed to yield an estimation of one or more parameters (output of the process) hidden in a usually nonlinear manner in the data. Herein, the process makes use of a cost (or objective) function.

This cost function gives a measure of the discrepancy between a measured (or simulated) field and a retrieval model of this field. The measured (or simulated) field (herein the SM) incorporates true values of $\mathbf{p}$, including those of $p_{K}$, whereas the retrieval model (RM) field incorporates trial values, designated by $P_{K}$, and more-or-less accurate values (with respect to their true counterparts in the data) of the other $p_{k} ; k \neq K$, designated by $P_{k}$. 
When, during the variation of $P_{K}$, the cost function attains a minimum, it is hoped that the trial value $P_{K}$ be as close as possible to the actual value of the parameter $p_{K}$.

During the inversion, $P_{K}$ is varied within the interval $\mathbb{I}$ in such a way as to approach the minimum of the cost function. At each step of the inversion (usually for diminishing $\mathbb{I}$ ), the set of $P_{k}$, including $P_{K}$, is designated by $\mathbf{P}$. Note that none of the parameters $P_{k}$ other than $P_{K}$ are varied during a given inversion. However, comparisons will be made of different inversions to retrieve $p_{K}$ incorporating different $\mathbf{Q}:=\mathbf{P} \cap P_{K}$.

A common cost function is of the least squares variety and is expressed by

$$
\kappa\left(P_{K}, \mathbf{Q}\right)=\frac{\int_{t_{d}}^{t_{f}}\left[U\left(P_{K} \cup \mathbf{Q}, x_{1}, 0, t\right)-U\left(\mathbf{p}, x_{1}, 0, t\right)\right]^{2} d t}{\int_{t_{d}}^{t_{f}}\left[U\left(\mathbf{p}, x_{1}, 0, t\right)\right]^{2} d t},
$$

wherein $U\left(\mathbf{p}, x_{1}, 0, t\right) ; t \in\left[t_{d}, t_{f}\right]$ is the data field and $U\left(P_{K} \cup \mathbf{Q}, x_{1}, O, t\right) ; t \in\left[t_{d}, t_{f}\right]$ the model field incorporating the trial value $P_{K}$ of the sought-for parameter $p_{K}$.

Actually, the signal only exists in discretized form, as $N_{t}$ samples in the window $\left[t_{d}, t_{f}\right]$, so as to lead to the alternate definition of the cost function

$$
\kappa\left(P_{K}, \mathbf{Q}\right)=\frac{\sum_{n=1}^{N_{t}}\left[U\left(P_{K} \cup \mathbf{Q}, x_{1}, 0, t_{n}\right)-U\left(\mathbf{p}, x_{1}, 0, t_{n}\right)\right]^{2}}{\sum_{n=1}^{N_{t}}\left[U\left(\mathbf{p}, x_{1}, 0, t_{n}\right)\right]^{2}} .
$$

wherein $t_{n}=t_{d}+(n-1) \varepsilon_{t}$ and $\varepsilon_{t}=\left(t_{f}-t_{d}\right) /\left(N_{t}-1\right)$.

\subsection{Minimization of the cost function}

The inverse problem is solved by minimization of the cost function. Assuming that it is the single parameter $p_{K}$ one wants to reconstruct, whose retrieval model counterpart is $P_{K}$, and that the minimum of the cost function is found for $P_{K}=\tilde{p}_{K}(\mathbf{Q}, \mathbb{I})$,

$$
\tilde{p}_{K}(\mathbf{Q}, \mathbb{I})=\arg \min _{P_{K} \in \mathbb{I}} \kappa\left(P_{K}, \mathbf{Q}\right) .
$$

The arg symbol in front of the min symbol means that the actual value of the minimum of $\kappa$ is irrelevant; rather it is the value of $P_{K}$ which produces this minimum that is the item of interest.

This formula suggests that:

- $\tilde{p}_{K}$ can be different from the true value $p_{K}$; in this case the inversion has been successful, but has produced a result that is fraught with error,

- $\tilde{p}_{K}$ depends on the search interval $\mathbb{I}:=\left[P_{K d}, P_{K f}\right]$; if the latter is too narrow, there might not exist a minimum of $\kappa$ therein, and if it is too wide, there might exist more than one minima therein (in which case it is usual, as is done herein, to retain the solution corresponding to the global minimum) and/or the precision of the retrieved parameter might be too low,

- $\tilde{p}_{K}$ depends on $\mathbf{Q}$, which means that differences between the parameter set $\mathbf{Q}$ employed in the retrieval model and the set $\mathbf{q}$ of the data simulation model will give rise to differences between $\tilde{p}_{K}$ and $p_{K}$; in fact it is of prime interest to see how the discordance between these two sets affects the precision of the retrieval of $p_{K}$. 
A relatively primitive, but nonetheless illustrative, way to carry out the inversion is by plotting the cost $\kappa$ on the $y$-axis versus $P_{K}$ on the $x$-axis; the value of $P_{K}$, for which $\kappa$ is visually found to be minimum, is $\tilde{p}_{K}$. A more quantitative method is to employ (as is done herein) a minimization scheme such as bisection to locate this abscissa. To get a more accurate estimation, the procedure is repeated for smaller intervals $\mathbb{I}$.

It should be stressed that the cost function may possess more than one (relative) minimum (Ogam et al., 2001) or no minimum, as may be the case in which there is discordance between $\mathbf{Q}$ and q. Moreover, the deepest (global) minimum may turn out not to correspond to the value of $P_{K}$ closest to $p_{K}$.

Thus, inversion does not necessarily lead to a solution, nor to a unique solution, nor to the correct solution.

\subsection{More on discordance and retrieval error}

Suppose that the parameter $P_{l} \in \mathbf{Q}$ is different from its counterpart $p_{l} \in \mathbf{q}$. Then the (relative percent) discordance between the two is:

$$
\delta_{l}:=\left(\frac{P_{l}-p_{l}}{p_{l}}\right) \times 100 .
$$

We shall be interested in particular in the effect of $10 \%$ discordances relative to one or more parameters on the error of the retrieval of another parameter.

This (relative percent) retrieval error is:

$$
\varepsilon_{K}=\left|\frac{\tilde{p}_{K}-p_{K}}{p_{K}}\right| \times 100,
$$

wherein $\tilde{p}_{K}$ is the retrieved value of $p_{k}$ by the inversion scheme.

All the material in the following five subsections applies to the configuration (assumed both in the data and the retrieval model): $a=0.1, t_{1}=0.0025 \mathrm{~s}, f_{0}=\omega_{0} / 2 \pi=100 \mathrm{rad}, t_{d}=0 \mathrm{~s}, t_{f}=0.03$ $\mathrm{s}, N_{t}=101$. The other physical and geometrical parameters relative to the data are given in table 1.

\begin{tabular}{cccc}
$p_{K}$ & parameter & value & units \\
\hline$p_{1}$ & $\rho$ & 2400 & $\mathrm{~kg} / \mathrm{m}^{3}$ \\
$p_{2}$ & $\Re \lambda$ & $12.3 \times 10^{9}$ & $\mathrm{~Pa}$ \\
$p_{3}$ & $\Im \lambda$ & 0 & $\mathrm{~Pa}$ \\
$p_{4}$ & $\Re \mu$ & $4 \times 10^{9}$ & $\mathrm{~Pa}$ \\
$p_{5}$ & $\Im \mu$ & $-0.1 \times 10^{9}$ & $\mathrm{~Pa}$ \\
$p_{6}$ & $x_{1}$ & 10 & $\mathrm{~m}$ \\
\hline
\end{tabular}

Table 1: Parameters of the set $\mathbf{p}$ employed in the data simulation model. These parameters will be retrieved, one at a time, by the inversion process.

Due to the fact that $p_{3}=\Im \lambda=0$, it was not possible to compute $\delta_{3}$ and $\varepsilon_{3}$. This means that we were unable to compute the retrieval error of $\Im \lambda$. 


\subsubsection{Illustration of the inversion process}

The following four figures (relative to the the retrieval of $\Re \mu$ ) illustrate how the inversion process was carried out, i.e.:

1- generate the data once and for all (red curve in the top panel of the figures);

2- for each $\mathbf{P}$ in the choice of $\mathbb{I}$ (in this example, for each $P_{1}$ in the interval $\left[P_{1 d}, P_{1 f}\right]$, the other $P_{k}$ of $\mathbf{P}$ being fixed), generate the response functions (black curves in the top panel of the figures);

3 - compute the cost function corresponding to each black curve and plot these cost functions as a function of $P_{K}$ (blue curve);

4- find the position ( $\arg \min$ ) of the minimum of the cost function;

5 - to increase the resolving power of this minimum position, diminish $\mathbb{I}$, while keeping constant or increasing the number of $P_{1}$ in this interval, and repeat operations 2-4 (second to fourth figures); 6 - the adopted $\widetilde{p}_{K}$ is the position of the minimum of the cost function in the last of the four figures.
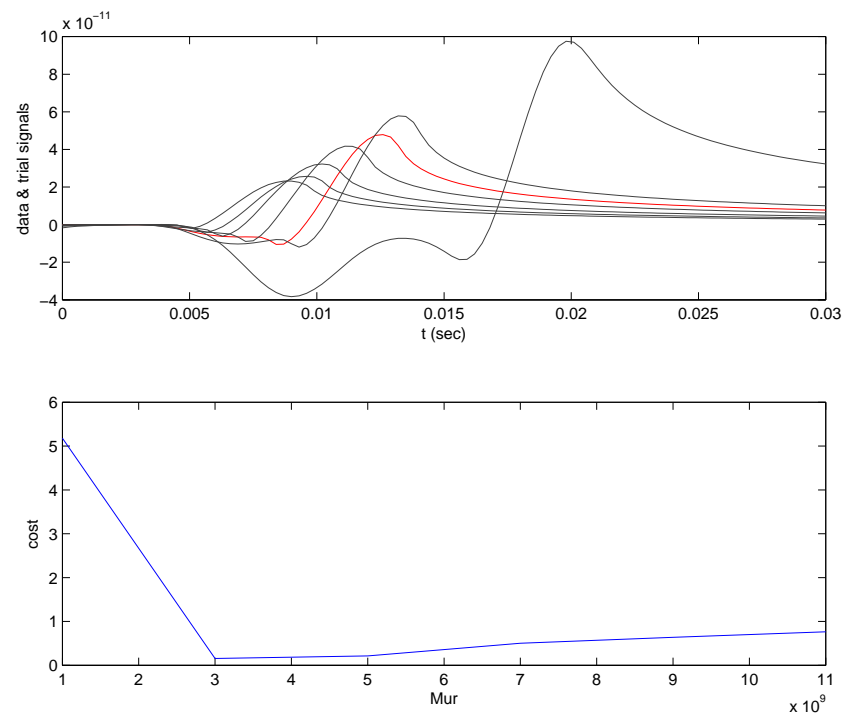

Figure 3: First iteration (for wide $\mathbb{I}$ ) in the inversion process for the retrieval of $\Re \mu$ for a single parameter $(\rho)$ discordance $\delta_{1}=-10$. Top panel: data (red) and various trial response curves (black). Bottom panel: cost function corresponding to the various trial responses.

The results of these operations, for various discordances, are given in tables 2-6. Each row in the tables represents the last in the series of (usually of the order of five) operations for diminishing I described in the above lines. 

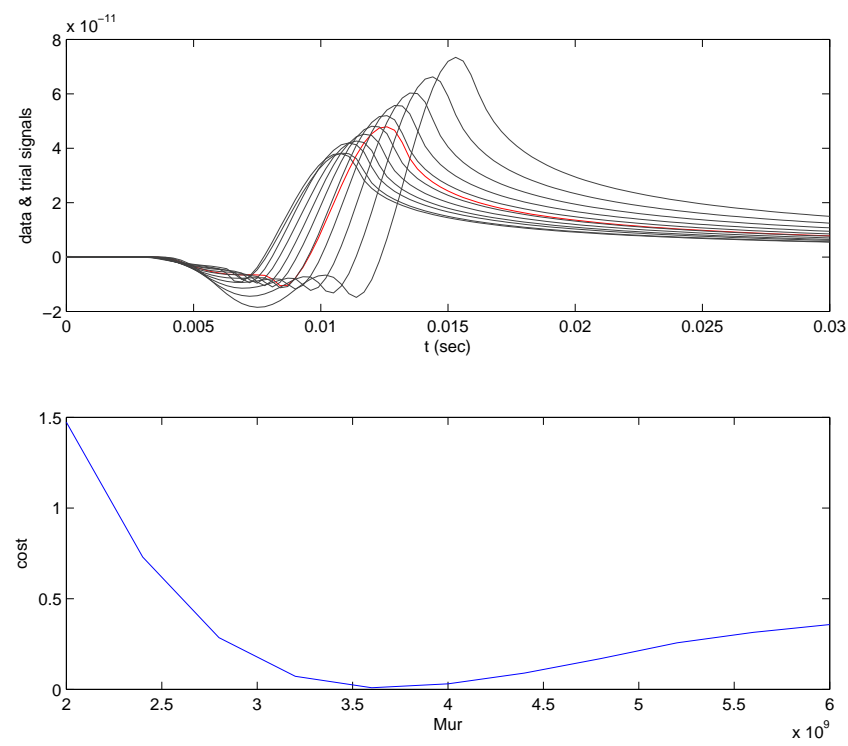

Figure 4: Second iteration (for narrower $\mathbb{I}$ ) in the inversion process for the retrieval of $\Re \mu$ for a single parameter $(\rho)$ discordance $\delta_{1}=-10$. Top panel: data (red) and various trial response curves (black). Bottom panel: cost function corresponding to the various trial responses.
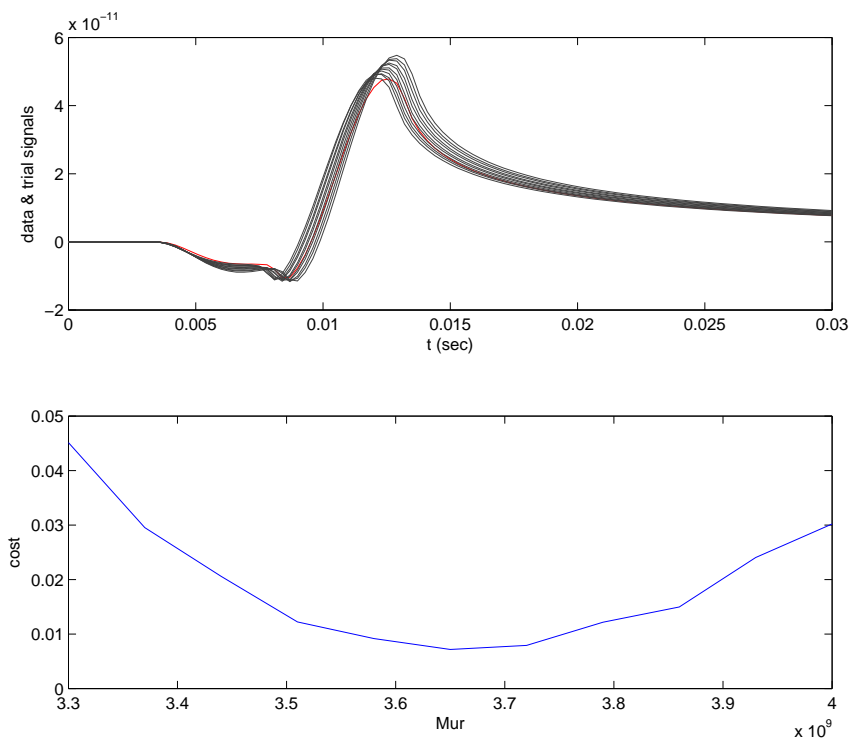

Figure 5: Third iteration (for even narrower $\mathbb{I}$ ) in the inversion process for the retrieval of $\Re \mu$ for a single parameter $(\rho)$ discordance $\delta_{1}=-10$. Top panel: data (red) and various trial response curves (black). Bottom panel: cost function corresponding to the various trial responses. 

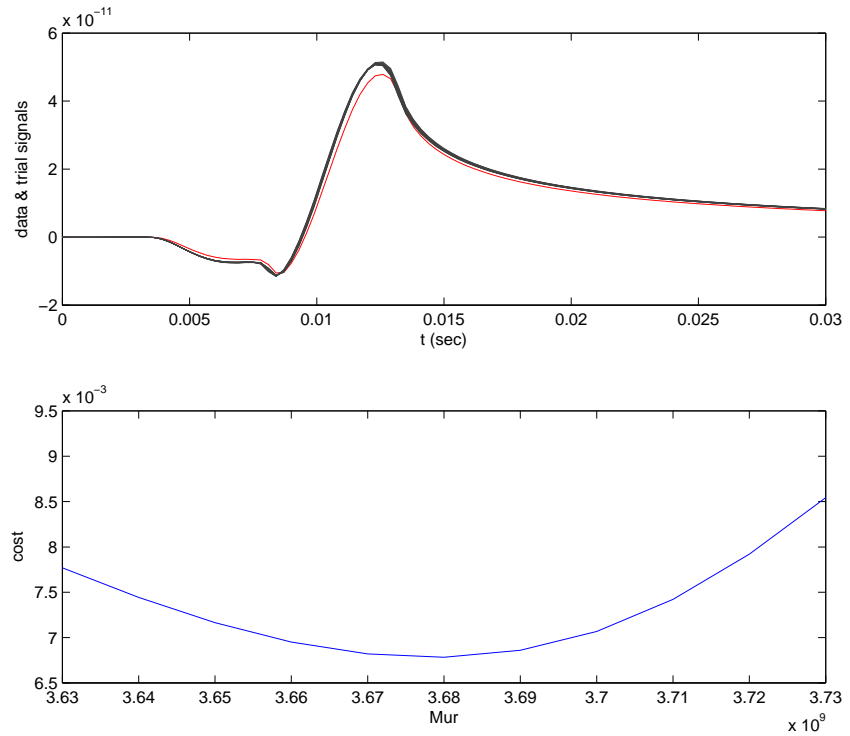

Figure 6: Fourth and final iteration (for narrowest I) in the inversion process for the retrieval of $\Re \mu$ for a single parameter $(\rho)$ discordance $\delta_{1}=-10$. Top panel: data (red) and various trial response curves (black). Bottom panel: cost function corresponding to the various trial responses. 


\subsubsection{Retrieval error of $\rho$}

Table 2 shows how the precision of the retrieval of $\rho$ depends on the discordances of the other parameters.

\begin{tabular}{ccccc}
$\delta_{2}$ & $\delta_{4}$ & $\delta_{5}$ & $\delta_{6}$ & $\varepsilon_{1}$ \\
\hline-10 & 0 & 0 & 0 & 0 \\
10 & 0 & 0 & 0 & 0.3 \\
0 & -10 & 0 & 0 & 8.3 \\
0 & 10 & 0 & 0 & 9.6 \\
0 & 0 & -10 & 0 & 0.1 \\
0 & 0 & 10 & 0 & 0 \\
0 & 0 & 0 & -10 & 25.0 \\
0 & 0 & 0 & 10 & 16.7 \\
\hline
\end{tabular}

Table 2: One discordance $\delta_{l}$ per inversion, and its effect on the error $\varepsilon_{1}$ of the retrieval of parameter $p_{1}=\rho$. The line $\delta_{2}=\delta_{4}=\delta_{5}=\delta_{6}=0$ (with $P_{3}=p_{3}$ ) is not shown in this table, but gave the expected result $\varepsilon_{1}=0$ corresponding to the inverse crime situation.

\subsubsection{Retrieval error of $\Re \lambda$}

Table 3 shows how the precision of the retrieval of $\Re \lambda$ depends on the discordances of the other parameters.

\begin{tabular}{ccccc}
$\delta_{1}$ & $\delta_{4}$ & $\delta_{5}$ & $\delta_{6}$ & $\varepsilon_{2}$ \\
\hline-10 & 0 & 0 & 0 & 18.7 \\
10 & 0 & 0 & 0 & 32.8 \\
0 & -10 & 0 & 0 & 69.9 \\
0 & 10 & 0 & 0 & 45.5 \\
0 & 0 & -10 & 0 & 1.6 \\
0 & 0 & 10 & 0 & 1.6 \\
0 & 0 & 0 & -10 & 31.7 \\
0 & 0 & 0 & 10 & 115.5 \\
\hline
\end{tabular}

Table 3: One discordance $\delta_{l}$ per inversion, and its effect on the error $\varepsilon_{2}$ of the retrieval of parameter $p_{2}=\Re \lambda$. The line $\delta_{1}=\delta_{4}=\delta_{5}=\delta_{6}=0$ (with $P_{3}=p_{3}$ ) is not shown in this table, but gave the expected result $\varepsilon_{2}=0$ corresponding to the inverse crime situation. 


\subsubsection{Retrieval error of $\Re \mu$}

Table 4 shows how the precision of the retrieval of $\Re \mu$ depends on the discordances of the other parameters.

\begin{tabular}{ccccc}
$\delta_{1}$ & $\delta_{2}$ & $\delta_{5}$ & $\delta_{6}$ & $\varepsilon_{4}$ \\
\hline-10 & 0 & 0 & 0 & 8.0 \\
10 & 0 & 0 & 0 & 6.8 \\
0 & -10 & 0 & 0 & 0.5 \\
0 & 10 & 0 & 0 & 0.5 \\
0 & 0 & -10 & 0 & 0 \\
0 & 0 & 10 & 0 & 0 \\
0 & 0 & 0 & -10 & 16.0 \\
0 & 0 & 0 & 10 & 19.4 \\
\hline
\end{tabular}

Table 4: One discordance $\delta_{l}$ per inversion, and its effect on the error $\varepsilon_{4}$ of the retrieval of parameter $p_{4}=\Re \mu$. The line $\delta_{1}=\delta_{2}=\delta_{5}=\delta_{6}=0$ (with $P_{3}=p_{3}$ ) is not shown in this table, but gave the expected result $\varepsilon_{4}=0$ corresponding to the inverse crime situation.

\subsubsection{Retrieval error of $\Im \mu$}

Table 5 shows how the precision of the retrieval of $\Im \mu$ depends on the discordances of the other parameters.

\begin{tabular}{ccccc}
$\delta_{1}$ & $\delta_{2}$ & $\delta_{4}$ & $\delta_{6}$ & $\varepsilon_{5}$ \\
\hline-10 & 0 & 0 & 0 & 35.0 \\
10 & 0 & 0 & 0 & 190.0 \\
0 & -10 & 0 & 0 & 20.0 \\
0 & 10 & 0 & 0 & 20.0 \\
0 & 0 & -10 & 0 & 90.0 \\
0 & 0 & 10 & 0 & 50.0 \\
0 & 0 & 0 & -10 & 500.0 \\
0 & 0 & 0 & 10 & 25.0 \\
\hline
\end{tabular}

Table 5: One discordance $\delta_{l}$ per inversion, and its effect on the error $\varepsilon_{5}$ of the retrieval of parameter $p_{5}=\Im \mu$. The line $\delta_{1}=\delta_{2}=\delta_{4}=\delta_{6}=0$ (with $P_{3}=p_{3}$ ) is not shown in this table, but gave the expected result $\varepsilon_{5}=0$ corresponding to the inverse crime situation. 


\subsubsection{Retrieval error of $x_{1}$}

Table 6 shows how the precision of the retrieval of $x_{1}$ depends on the discordances of the other parameters.

\begin{tabular}{ccccc}
$\delta_{1}$ & $\delta_{2}$ & $\delta_{4}$ & $\delta_{5}$ & $\varepsilon_{6}$ \\
\hline-10 & 0 & 0 & 0 & 5.4 \\
10 & 0 & 0 & 0 & 4.7 \\
0 & -10 & 0 & 0 & 0.3 \\
0 & 10 & 0 & 0 & 0.2 \\
0 & 0 & -10 & 0 & 4.6 \\
0 & 0 & 10 & 0 & 4.6 \\
0 & 0 & 0 & -10 & 0 \\
0 & 0 & 0 & 10 & 0 \\
\hline
\end{tabular}

Table 6: One discordance $\delta_{l}$ per inversion, and its effect on the error $\varepsilon_{6}$ of the retrieval of parameter $p_{6}=x_{1}$. The line $\delta_{1}=\delta_{2}=\delta_{4}=\delta_{5}=0$ (with $\left.P_{3}=p_{3}\right)$ is not shown in this table, but gave the expected result $\varepsilon_{1}=0$ corresponding to the inverse crime situation.

\subsubsection{Comments on the tables relative to the retrieval errors resulting from the dis- cordances}

What these tables reveal are that:

a) the retrieval errors of the various constitutive parameters are far from negligible, even for a discordance of a single parameter that is as small as $10 \%$;

b) the retrieval errors of $\rho, \Re \lambda, \Re \mu, \Im \mu$ are generally largest for model discordance pertaining to $x_{1}$

c) the retrieval errors of $\Re \lambda$ are largest for $\Re \mu$ model discordances and somewhat smaller for $\rho$ model discordances;

d) the sensitivity of the retrieval of the other parameters to discordance of $\Im \mu$ is weak; this is probably related to the fact that the retrieval error of $\Im \mu$ is very large for most model discordances; e) the retrieval error of $x_{1}$ is acceptably small for all model discordances.

\section{Conclusion}

The data, which is processed in the inversion scheme, was obtained by numerical simulation. The underlying physical-mathematical model thereof is a supposedly-rigorous solution (expressed by a double integral) of the boundary value problem of continuum elastodynamics in a linear, homogeneous, isotropic hysteretically-damped solid occupying a half space and solicited by a strip load on its boundary.

The retrieval model employed the same supposedly rigorous physical-mathematical solution, as well as its numerical translation.

The numerics were of a very basic variety in both the data simulation and retrieval models: Simpson quadrature for the first $(\xi)$ integral and Simpson quadrature for the second $(f)$ integral. 
Thus, the inverse crime was committed when all the entries in the set $\mathbf{P}$ of parameters of the retrieval model were identical to their counterparts in the set $\mathbf{p}$ of the data simulation model.

However, in real life, this situation is nearly impossible to attain, due to an imprecise a priori knowledge of at least one of the not-to-be-retrieved parameters in $\mathbf{P}$, and it is this imprecision which leads to a retrieval error in the other parameter(s). In fact, it was observed that parameter discordance was the cause of the occasional occurrence, during the inversion, of more than one minima in the cost function. We always chose the solution corresponding to the global minimum, but this procedure is admittedly ambiguous. It would be interesting to find out if these local minima disappear when more (and perhaps of a different nature) data is processed in the inversion scheme.

This imprecision, for a $\pm 10 \%$ discordance between a given entry in $\mathbf{P}$ and its counterpart in $\mathbf{p}$, was evaluated for the retrieval, one at a time, of the five mechanical descriptors and single geometrical descriptor of the source position.

The retrieval errors of the various constitutive parameters were found to be far from negligible, even for a discordance of a single parameter that is as small as $10 \%$; this finding means that inversion results pertaining to mechanical parameter retrieval should be treated with caution, especially if no mention is made of the underlying imprecision of the parameters that are fixed a priori (and considered to be "known") during the inversion. Note that if the chosen (rather small $10 \%$, considering that parameter uncertainty can easily attain $100 \%$ for certain parameters in field practice) discordance had been larger, the message this investigation conveys would have been less vivid.

It was shown that the retrieval errors of the mechanical descriptors are largest for discordance of the source position $x_{1}$ and that it is nearly-impossible to obtain a reliable retrieval of the imaginary part of $\Im \mu$ for $\pm 10 \%$ discordance of any of the other mechanical descriptors or of $x_{1}$.

On the other hand, it was shown that a $\pm 10 \%$ discordance of the mechanical descriptors resulted in reasonably-small error in the retrieval of $x_{1}$, which is an encouraging result for source location retrieval (although it will have to be substantiated for more complex environments than the one considered herein (Michelini \& Lomax, 2004)).

If more than one parameters are subject to $\pm 10 \%$ discordances, and/or the discordances are larger than the ones considered herein, it is expected that the error of the retrieved mechanical parameters will be unacceptably large. It might be possible to reduce these errors by processing more (and perhaps of a different nature) data.

In this connection, it would be of great interest to replace the simulation model by one that is fundamentally different, at least as concerns its numerical aspects (e.g., resolve the time domain boundary value problem in direct manner by a finite difference or finite element scheme such as is in (Virieux, 1986) or (Mesgouez, 2005)). The reason for doing this would be to show that discordances between the numerical aspects of the RM and SM lead to retrieval errors that are of similar nature to those resulting from discordances between $P$ of the RM and $p$ of the SM. Moreover, retrieval errors would supposedly exist resulting from a discordance between the prediction of the RM and real data (even if the latter is generated in a laboratory environment).

A natural extension of this study is to generalize the solicitation to include a horizontal component, and to collect and incorporate horizontal displacement component response in the data sample which is analyzed during the inversion. Moreover, it might be useful, as in field practice, to collect and process data at multiple receiver locations on the ground.

A necessary generalization of this investigation is the retrieval of the viscoelastic parameters of a layer (or multilayer structure) overlying a homogeneous viscoelastic half space and the treatment of 
the corresponding axisymmetric problem (i.e, a uniform circular patch solicitation on the ground). 


\section{References}

[1] Aki K. and Richards P.G., Quantitative Seismology, Theory and Methods, Vol. II, Freeman, New York (1980).

[2] Apsel R.J and Luco J.E., On the Green's functions for a layered half-space. Part II, Bull.Seism.Soc.Am., 73, 931-951 (1983).

[3] Billings S.D., Kennett B.L.N. and Sambridge M.S., Hypocentre location: genetic algorithms incorporating problem-specific information, Geophys.J.Int., 118, 693-706 (1994).

[4] Bodet L., Limites théoriques et expérimentales de l'interprétation de la dispersion des ondes de Rayleigh: apport de la modélisation numérique et physique, Thesis, Université de Nantes, Nantes (2005).

[5] Buchanan J.L., Gilbert R.P. and Ou M-J.Y., Recovery of the parameters of cancellous bone by inversion of effective velocities, and transmission and reflection coefficients, Inv.Probs., 27, 125006, doi:10.1088/0266-5611/27/12/125006 (2011).

[6] Buchanan J.L., Gilbert R.P., Wirgin A. and Xu Y., Depth sounding: an illustration of some of the pitfalls of inverse scattering problems, Math.Comput.Model., 35, 1315-1354 (2002).

[7] Chen X.C. and Zhang H., An efficient method for computing Green's functions for a layered half-space at large epicentral distances, Bull.Seism.Soc.Am., 91, 858-869 (2001).

[8] Chotiros N.P., An inversion for Biot parameters in water-saturated sand, J.Acoust.Soc.Am., $112,1853-1868$ (2002).

[9] Colladon J.D. and Sturm J.K.F., Mémoire sur la Compression des Liquides, Annal.Chim.Phys., Série 2,36, part IV, La vitesse du son dans les liquides, 236-257 (1827).

[10] Colton D. and Kress R., Inverse Acoustic and Electromagnetic Scattering Theory, Springer, Berlin (1992).

[11] De Barros L., Dietrich M. and Valette B., Full waveform inversion of seismic waves reflected in a stratified porous medium, Geophys.J.Int., 182, 1543-1556 (2010).

[12] Dupuy B., Propagation des ondes sismiques dans les milieux multiphasiques hétérogènes: modélisation numérique, sensibilité et inversion des paramètres poroélastiques, Thesis, Université de Grenoble, Grenoble (2011).

[13] Eringen A.C. and Suhubi E., Elastodynamics, vol. 2: Linear Theory, Academic Press, New York, 614-629 (1975).

[14] Foti S., Comina C., Boiero D. and Socco L.V., Non-uniqueness in surface-wave inversion and consequences on seismic site response analyses, Soil Dynm.Earthqu.Engrg., 29, 982-993 (2009).

[15] Fu C.Y., Studies on seismic waves: III. Propagation of elastic waves in the neighborhood of a free boundary, Geophys., 12, 57-71 (1947). 
[16] Groby J.-P., Modélisation de la propagation des ondes élastiques générées par un séisme proche ou éloigné à l'intérieur d'une ville, Thèse de l'Université Aix-Marseille II, Marseille, (2005).

[17] Groby J.-P. and Wirgin A., Two-dimensional ground motion at a soft viscoelastic layer/hardsubstratum site in response to SH cylindrical seismic waves radiated by deep and shallow line sources-I. Theory, Geophys.J.Int., 163, 165191 (2005).

[18] Jones D.V. and Petyt M., Ground vibration in the vicinity of a strip: a two-dimensional halfspace model, J.Sound Vibr., 147, 155-166 (1991).

[19] Kausel E., Fundamental Solutions in Elastodynamics-A Compendium, Cambridge Univ. Press, Cambridge (2006).

[20] Kikuchi M. and Kanamori H., Inversion of complex body waves, Bull.Seism.Soc.Am., 72, 491506 (1982).

[21] Lai C.G., Rix G.J., Foti S. and Roma V., Simultaneous measurement and inversion of surface wave dispersion and attenuation curves, Soil Dynam.Earthquake Engrg., 22, 923- 930 (2002).

[22] Ma C.C. and Lee G.-S., Transient elastic waves propagating in a multi-layered medium subjected to in-plane dynamic loadings II. Numerical calculation and experimental measurement, Proc.R.Soc.Lond. A 456, 1375-1396 (2000).

[23] Mesgouez A., Etude numérique de la propagation des ondes mécaniques dans un milieu poreux en régime impulsionnel, Thesis, Université d'Avignon et des pays de Vaucluse, Avignon (2005).

[24] Mesgouez A. and Lefeuve-Mesgouez G., Transient solution for multilayered poroviscoelastic media obtained by an exact stiffness matrix formulation, Int.J.Numer.Analyt.Meth.Geomech., 33, 1911-1931 (2009).

[25] Michelini A. and Lomax A., The effect of velocity structure errors on double-difference earthquake location, Geophys.Res.Lett., 31, L09602, doi:10.1029/2004GL019682 (2004).

[26] Molenkamp F. and Smith I.M., Hysteretic and viscous material damping, Int.J.Numer.Anal.Meth.Geomech., 4, 293-311 (1980).

[27] Mora P., Nonlinear two-dimensional elastic inversion of multioffset seismic data, Geophys., 52, 1211-1228 (1987).

[28] Ogam E., Scotti T. and Wirgin A., Non-ambiguous boundary identification of a cylindrical object by acoustic waves, C.R.Acad.Sci.Paris IIb, 239, 61-66 (2001).

[29] Park J. and Kausel E., Impulse response of elastic half-space in the wave number-time domain, J.Engrg.Mech. ASCE, 130, 1211-122 (2004).

[30] Pratt G., Frequency-domain elastic wave modeling by finite differences: a tool for crosshole seismic imaging, Geophys., 55, 626-632 (1990).

[31] Pratt G., Seismic waveform inversion in the frequency domain, Part 1; theory and verification in a physical scale model, Geophys., 64, 888-901 (1999). 
[32] Ringdal F. and Kennett B.L.N. (eds.), Monitoring the comprehensive Nuclear-Test-Ban Treaty: Source Location, Birkhauser, Basel (2001).

[33] Sacks P. and Symes W., Recovery of the elastic parameters of a layered half-space, Geophys.J.R.Astron.Soc., 88, 593-620 (1987).

[34] Scotti T. and Wirgin A., Reconstruction of the three mechanical material constants of a lossy fluid-like cylinder from low-frequency scattered acoustic fields, C.R.Méca., 332, 717-724 (2004).

[35] Stam H. J., The two-dimensional elastodynamic distributed surface load problem, Geophys., 55, 1047-1056 (1990).

[36] Sun R. and McMechan G.A., Full-wavefield inversion for wide-aperture, elastic, seismic data, Geophys.J.Int., 111, 1-10 (1992).

[37] Tarantola A., A strategy for nonlinear elastic inversion of seismic reflection data, Geophys., 51, 1893-1903 (1986).

[38] Thurber C.H. and Rabinowitz N. (eds.), Advances in Seismic Event Location, Springer, Berlin (2000).

[39] Valentine A.P. and Woodhouse J.H., Reducing errors in seismic tomography: combined inversion for sources and structure, Geophys.J.Intl., 180, 847-857 (2010).

[40] Virieux J., P-SV wave propagation in heterogeneous media: velocity-stress finite-difference method, Geophys.,51, 889-900 (1986).

[41] Virieux J. and Operto S., An overview of full-waveform inversion in exploration geophysics, Geophys.,74, WCC127-WCC152 (2009).

[42] Wirgin A., The inverse crime, http://arxiv.org/abs/math-ph/0401050 (2004).

[43] Xia J., Miller R.D. and Park C.B., Estimation of near-surface shear-wave velocity by inversion of Rayleigh waves, Geophys., 64, 691-700 (1999).

[44] Xu P.-C. and Mal A.K., Calculation of the inplane Green's functions for a layered viscoelastic solid, Bull.Seism.Soc.Am., 77, 1823-1837 (1987).

[45] Zhang S.X. and Chan L.S. Possible effects of misidentified mode number on Rayleigh wave inversion, J.Appl.Geophys. 53, 1729 (2003). 